\title{
Determining the Electron Density and Volume Expansion at Grain Boundaries Using Electron Energy-Loss Spectroscopy
}

\author{
Proloy Nandi ${ }^{1}$, Eric Hoglund ${ }^{1}$, Xiahan Sang $^{2}$, Raymond R. Unocic ${ }^{2}$ and James M. Howe ${ }^{1}$ \\ 1. Dept. of Materials Science \& Engineering, University of Virginia, Charlottesville, VA 22904, USA \\ 2. Center for Nanophase Materials Sciences, Oak Ridge National Laboratory, Oak Ridge, TN 37831, USA
}

Grain boundary (GB) energy is an important parameter in determining the microstructure and properties of materials. Seeger et al. [1] first proposed a dependence of the GB energy in metals on a volume expansion $(\delta V)$ normal to the boundary with a corresponding decrease in the electron density $(n)$ at the GB. In this work, valence electron energy loss spectroscopy (VEELS) and extended energy-loss fine structure (EXELFS) analysis were used to examine fundamental GB properties such as the electron density and volume expansion, respectively.

In the low-loss range $(0-50 \mathrm{eV})$ of an EELS spectrum, plasmons peaks result from losses due to the collective oscillations of valance electrons. According to the Drude model [2], the loss function of a volume plasmon is proportional to the imaginary component $\mathfrak{I}(-1 / \varepsilon)$, where $\varepsilon$ is the dielectric function, and $\varepsilon$ is correlated to the plasmon energy $\left(E_{p}\right)$, which is a function of the electron density $n$. In addition to the bulk plasmon mode, plasmon excitations can occur at interfaces and the loss function for an interface is proportional to $\mathfrak{J}\left(-\frac{2}{\varepsilon_{1}+\varepsilon_{2}}+\frac{1}{\varepsilon_{1}}\right)$, where the first term describes a mode due to the two different material dielectric constants, and the second term describes the begrenzungs effect. These relationships make it possible to determine the difference in $n$ between a matrix and GB by measuring $E_{p}$ in the two regions. Additionally, EXELFS modulations in an EELS spectrum provide information about nearest neighbor (n.n.) distances between atoms [3] and this can be used to probe changes in $\delta V$ at a GB.

Scanning transmission electron microscope (STEM) foils were prepared from Al bi-crystals (99.998\% purity) containing a medium-energy $\left(380-400 \mathrm{~mJ} / \mathrm{m}^{2}\right) 52.7^{\circ}<110>$ tilt GB. VEELS experiments were performed on a Hitachi $2700 \mathrm{C}$ aberration-corrected STEM operating at $200 \mathrm{kV}$ with $\sim 0.35 \mathrm{eV}$ energy spread and a Nion UltraSTEM 100 operating at $100 \mathrm{kV}$ with $\sim 0.30 \mathrm{eV}$ energy spread. Plasmon peaks from individual EELS spectra were fitted with a Gaussian function to determine $E_{p}$ to an accuracy of better than $0.05 \mathrm{eV}$. EXELFS experiments were performed using a FEI Titan 80-300 (S)TEM operating at $300 \mathrm{kV}$ with a Schottky field-emission gun. EELS spectra of the $\mathrm{Al} \mathrm{L}_{2,3}(73 \mathrm{eV})$ and $\mathrm{L}_{1}(118 \mathrm{eV})$ edges were collected using a spectrometer dispersion of $0.2 \mathrm{eV} /$ channel in the image-coupled mode to accurately place the $\sim 0.2 \mathrm{~nm}$ probe on the GB, as shown by the blue dots in Fig. 1a. Nearest-neighbor distances were calculated from radial distribution functions (RDF) obtained from the EXELFS modulations.

Fig. 1a shows a HAADF STEM image and corresponding diffraction pattern (Fig. 1b) of the edge-on GB. A line scan $15 \mathrm{~nm}$ long with a step increment of $0.1 \mathrm{~nm}$ was taken across the GB, as indicated by the red line in Fig. 1a. The corresponding EELS spectrum image (SI) aligned with respect to the zeroloss peak (ZLP) is shown in Fig. 2a. The plasmon peak position shifts to lower energies at the GB, as 
seen by the region enclosed between the horizontal lines in Fig. 2a. A plot of $E_{p}$ across the GB is shown in Fig. 3, where a Voigt function (red curve) was fitted to the data to quantify the behavior. These results show that $E_{p}$ decreases by approximately $0.05 \mathrm{eV}$ at the $\mathrm{GB}$ (i.e., from 14.92 to $14.87 \mathrm{eV}$ in Fig. 3 ), which corresponds to a decrease in $n$ of about $10 \mathrm{e}^{-} / \mathrm{nm}^{3}$. This decrease extends a distance of roughly $2.4 \mathrm{~nm}$ across the GB, which is several times wider than the width of the GB region estimated from the HAADF image in Fig. 1a. Fig. $2 b$ shows the GB plasmon after deconvolution, where the interfacial mode of the GB plasmon causes additional energy-loss below $15 \mathrm{eV}$, and less loss of the bulk mode above $15 \mathrm{eV}$ that is attributed to the begrenzungs effect. Fig. 4 shows the RDF results, where an increase in the first n.n. spacing at the GB (red curve) of $\sim 0.07$ Angstrom was found. Thus, the VEELS and EXELF results demonstrate that $n$ decreases and $\delta V$ increases at the GB [1], and further effort is currently underway to quantify these effects as they relate to GB energy [4].

\section{References:}

[1] A.Seeger et al, , Acta metallurgica 7 (1959) p. 495.

[2] R.F. Egerton, "Electron energy-loss spectroscopy in the electron microscope, $3^{\text {rd }}$ Ed." (Springer, New York) 2011.

[3] M. Sarikaya et al., Micron 27 (1996) p. 449.

[4] This research was supported by NSF Grant DMR-1106230 and the VPR Office at UVA. The authors thank Prof. D. Molodov for proving the Al bi-crystals, and Drs. Eric Stach and Dong Su for help with research conducted in part at CFN-BNL supported by the U.S. DOE-BES under Contract DE-SC00112704, and research supported as part of a user proposal at ORNL-CNMS, which is a U.S. DOE Office of Science User Facility (XS and RRU).

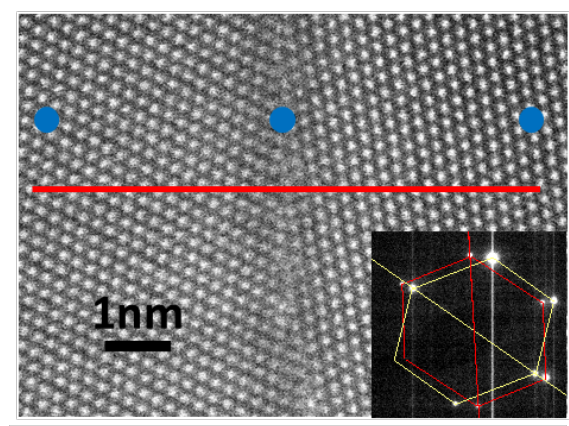

Figure 1. HAADF image and diffraction pattern of $52.7^{\circ}<110>$ tilt GB.

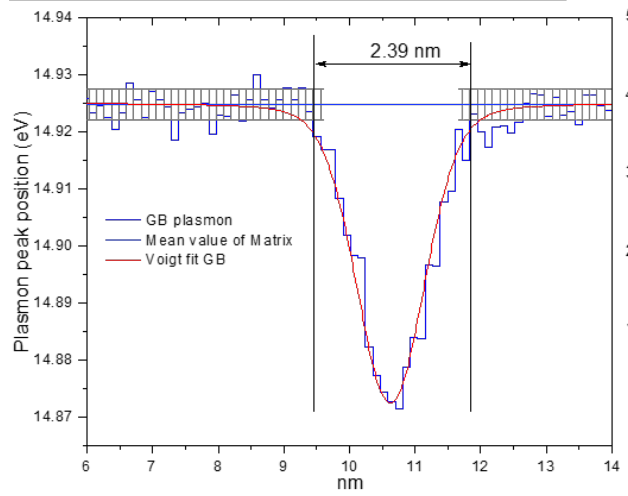

Figure 3. Volume plasmon peak shift across the GB.

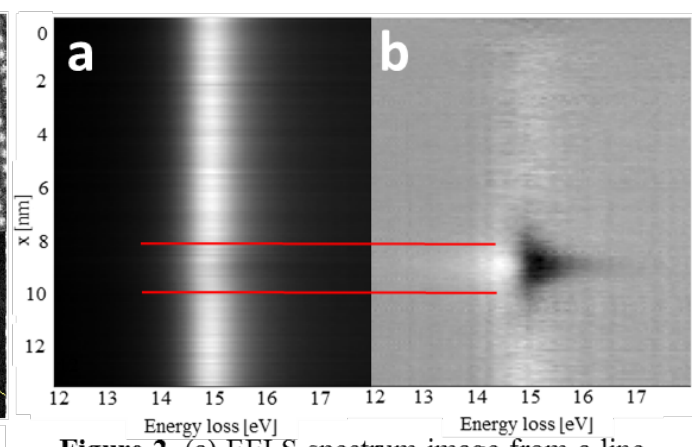

Figure 2. (a) EELS spectrum image from a line scan across the GB, aligned wrt. ZLP (b) deconvoluted GB plasmon.

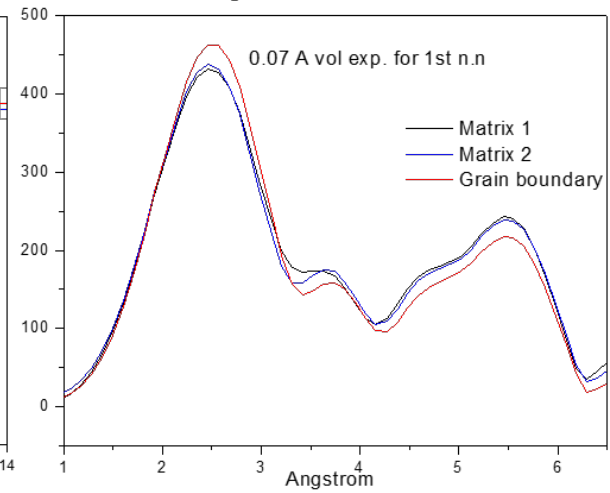

Figure 4. RDF obtained by Fourier transformation of the EXELFS modulations. 